Short communication

\title{
Identification of elastic properties of human patellae using micro-finite element analysis
}

\author{
Adeliya Latypova $^{\mathrm{a}}$, Ghislain Maquer ${ }^{\mathrm{b}}$, Kaliyaperumal Elankumaran ${ }^{\mathrm{c}}$, Dieter Pahr ${ }^{\mathrm{c}}$, \\ Philippe Zysset ${ }^{\mathrm{b}}$, Dominique P. Pioletti ${ }^{\mathrm{a}}$, Alexandre Terrier ${ }^{\mathrm{a}, *}$ \\ a Laboratory of Biomechanical Orthopedics, EPFL, Lausanne, Switzerland \\ ${ }^{\mathrm{b}}$ Institute for Surgical Technology and Biomechanics, UniBe, Bern, Switzerland \\ ' Institute of Lightweight Design and Structural Biomechanics, TUWien, Vienna, Austria
}

\section{A R T I C L E I N F O}

\section{Article history:}

Accepted 26 July 2016

\section{Keywords:}

Patella

Bone mechanics

Trabecular anisotropy

Finite element analysis

\begin{abstract}
A B S T R A C T
Current homogenized finite element (hFE) models of the patella lack a validated material law and mostly overlook trabecular anisotropy. The objective of this study was to identify the elastic constants of patellar trabecular bone. Using $\mu \mathrm{CT}$ scans of 20 fresh-frozen cadaveric patellae, we virtually extracted 200 trabecular cubes ( $5.3 \mathrm{~mm}$ side length). Bone volume fraction and fabric tensor were measured. The elastic constants were identified from six independent load cases using micro finite element ( $\mu \mathrm{FE})$ analyses. Both anisotropic and isotropic material laws were considered. The elastic constants were validated by comparing stiffness, strain and stress between hFE and $\mu \mathrm{FE}$ predictions of 18 patellar sections and six load cases. The hFE section models were built from $\mu \mathrm{CT}$ (anisotropic law) and CT (isotropic law) scans. The homogenized anisotropic model induced less error $(13 \pm 5 \%)$ in the global stiffness prediction than the isotropic one $(18 \pm 6 \%)$, and less error in the prediction of local apparent strain, stress, and strain energy, compared to the isotropic one. This validated hFE model could be used for future applications, either with the anisotropic constants, or with the isotropic ones when the trabecular fabric is unavailable.

(c) 2016 Elsevier Ltd. All rights reserved.
\end{abstract}

\section{Introduction}

Currently, the existing homogenized finite element (hFE) models of patella rely on material laws identified not on the patellar bone, but, for example, femur or vertebra (Fitzpatrick et al., 2011; Ho et al., 2014; Takahashi et al., 2012). Furthermore, the anisotropy of the trabecular bone, shown to be crucial for predicting its elastic properties on various anatomical sites (Maquer et al., 2015), is completely overlooked.

Abrupt changes of main trabecular orientations (Raux et al., 1975) and the small size of the patella make it difficult to extract samples of appropriate dimensions for biomechanical testing and complicate accurate experimental measurements (Lammentausta et al., 2006). In such cases, micro finite element ( $\mu \mathrm{FE}$ ) modeling based on high-resolution $\mu \mathrm{CT}$ reconstruction is a common alternative to in vitro mechanical testing (Pistoia et al., 2002; van Rietbergen and Ito, 2015; Wolfram et al., 2010). This method avoids preparation- and damage-related artefacts and restriction

\footnotetext{
* Correspondence to: Laboratory of Biomechanical Orthopedics, Ecole Polytechnique Fédérale de Lausanne, Station 19, 1015 Lausanne, Switzerland. Fax: +41216938660.

E-mail address: alexandre.terrier@epfl.ch (A. Terrier).
}

regarding the number of load-cases used to assess the elastic constants. Besides, the influence of any bony feature observable on $\mu \mathrm{CT}$ images can be reflected in the analysis.

Therefore, the aim of this study was to identify and validate elastic constants of the patellar bone specifically. The anisotropic constitutive law based on morphology-elasticity relationship and alternative isotropic law based on bone volume fraction were considered. Identification of homogenized models was performed by means of $\mu \mathrm{FE}$ simulated mechanical tests of $\mu \mathrm{CT}$ scanned cadaveric patellae. To validate the identified parameters, predictions of hFE models build from $\mu \mathrm{CT}$ and CT scans of cadaveric patellar sections were compared to $\mu \mathrm{FE}$ predictions of the same patellae.

\section{Materials and methods}

\section{1. $\mu C T$ and $C T$ imaging}

Twenty fresh-frozen cadaveric patellae (12 male, 8 female; age range 15-93, mean age $67 \pm 17$ ) after thawing at room temperature overnight and bubble removal, were scanned with a $\mu \mathrm{CT}$ (Skyscan 1076, Bruker microCT, Kontich, Belgium) in a saline soaked gauze with the following scanning settings: $18.3 \mu \mathrm{m}$ resolution, $80 \mathrm{kV} / 120 \mu \mathrm{A}, 540 \mathrm{~ms}$ exposure time, $1 \mathrm{~mm}$ aluminum filter, $0.2^{\circ}$ rotation step. The patellae were then CT scanned (Discovery 110 CT750 HD, GE Healthcare, Milwaukee, USA) with a resolution of $0.39 \times 0.39 \times 0.625 \mathrm{~mm}^{3}$. The $\mu \mathrm{CT}$ 
images were downscaled to $36.6 \mu \mathrm{m}$ resolution and segmented using a single level threshold algorithm (Ridler and Calvard, 1978). Image pre-processing was done in Medtool (www.dr-pahr.at).

\subsection{Identification of the material laws}

The following constitutive law was considered (Zysset, 2003; Zysset and Curnier, 1995):

$E_{i}=E_{0} \rho^{k}\left(m_{i}^{2}\right)^{l}, \frac{E_{i}}{\nu_{i j}}=\frac{E_{0}}{\nu_{0}} \rho^{k}\left(m_{i} m_{j}\right)^{l}, G_{i j}=G_{0} \rho^{k}\left(m_{i} m_{j}\right)^{l}, \forall i \neq j=1,2,3$,

where $E_{i}, v_{i j}$, and $G_{i j}$ are engineering constants, $E_{0}, \nu_{0}, G_{0}, k, l$ are model parameters, $\rho$ is the bone volume fraction, and $m_{i}$ are the normalized eigenvalues of the second-order fabric tensor $\boldsymbol{M}$ (Cowin, 1985). The isotropic case was based on the same relationship with $\boldsymbol{M}$ equal to the identity tensor $\boldsymbol{I}$.

To identify the model parameters, simple mechanical tests were conducted via $\mu \mathrm{FE}$ on 200 trabecular cubes (5.3 mm side length), virtually extracted from $\mu \mathrm{CT}$ scans of all patellae. Cubes were visually checked to have relatively homogeneous bone distribution within volume, without abrupt change in trabecular direction, and a sufficient amount of trabecular on the sides to provide proper load transition (Fig. 1). Bone volume fraction $(\rho)$, defined as bone volume over the total tissue volume, fabric tensor $\boldsymbol{M}$, established through mean intercept length (MIL) (Laib et al., 1998; Whitehouse, 1974), and the degree of anisotropy (DA), defined as a ratio between maximum and minimum eigenvalues of fabric tensor (Hildebrand et al., 1999), were measured for each cube. All cubes were converted into $\mu \mathrm{FE}$ models with linear hexahedral elements (about 9 million degrees of freedom) following a linear elastic law ( $E=12 \mathrm{GPa}, \boldsymbol{\nu}=0.3$ ) for bone tissue (Wolfram et al., 2010). Three compression and three shear load cases were applied to each cube under kinematic uniform boundary conditions (KUBC) (Pahr and Zysset, 2008). The full stiffness tensor $\left(\mathbf{C}_{\mu F E}\right.$ aniso $)$ of each cube was obtained by averaging $\mu \mathrm{FE}$ local strain and stress predictions. The orthotropic approximation $\left(\mathbf{C}_{\mu F E \text { ortho }}\right)$ of the stiffness tensor was considered for further analysis. The norm error associated with this assumption was calculated by

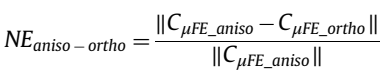

Model parameters were identified by fitting the material law (1) to components of $\mu \mathrm{FE}$ stiffness tensor, using multi linear regressions in logarithmic scale. The same procedure was repeated for the isotropic model. Pre- and post-processing were done in Medtool, and $\mu \mathrm{FE}$ simulations were performed using ParFE (parfe.source forge.net).

\subsection{Validation of the material laws}

To validate the material laws, $\mu \mathrm{FE}$ and hFE predictions of cuboid sections $\left(15 \times 22 \times 16 \mathrm{~mm}^{3}\left( \pm 8 \mathrm{~mm}^{3}\right)\right)$ of 18 out of 20 patellae were compared. One patella was excluded due to bone defects (probably metastases), and for one section $\mu \mathrm{FE}$ simulations did not converge. The sections were extracted from $\mu \mathrm{CT}$ scans by cropping the superior-inferior and medial-lateral sides, and embedding the anterior and posterior sides (Fig. 1). The embedding was modeled as linear elastic material $(E=1400 \mathrm{MPa}, \boldsymbol{\nu}=0.3)$. Three tension and three shear KUBC load cases were simulated. Each $\mu \mathrm{FE}$ model had approximately 350 million degrees of freedom. The $\mu \mathrm{FE}$ simulations were performed using ParOSol (bitbucket.org/Elanku maran/parosol-tu-wien). The hFE model meshes were created based on the same cropped $\mu \mathrm{CT}$ images downscaled to $0.54 \mathrm{~mm}$ voxel size. Each voxel was converted to a linear hexahedral element. Material properties of each bone element of the anisotropic model were automatically assigned by Medtool from original segmented $\mu \mathrm{CT}$ images, according to bone volume fraction and fabric (MIL) measured in 5.3-mm-diameter spherical volumes positioned in nodes of the $\mu \mathrm{CT} 2.0-\mathrm{mm}$ side-length background grid (Pahr and Zysset, 2009). In case of the isotropic model, the bone volume fraction was estimated from Hounsfield units (HU) of CT calibrated images (Latypova et al., 2016):

$\rho=1.0614 \times \mathrm{BMD}-0.0573, \mathrm{BMD}=\mathrm{HU} / 1510$

The same parameters for background grid and spherical volumes were used. Each hFE model had approximately $9 \times 10^{4}$ degrees of freedom. The hFE simulations were performed with Abaqus/Standard (Simulia, Providence, RI, USA).

To compare global $\mu \mathrm{FE}$ and hFE predictions, the stiffness matrices of the whole sections were calculated through the averaged strain and stress tensors and the
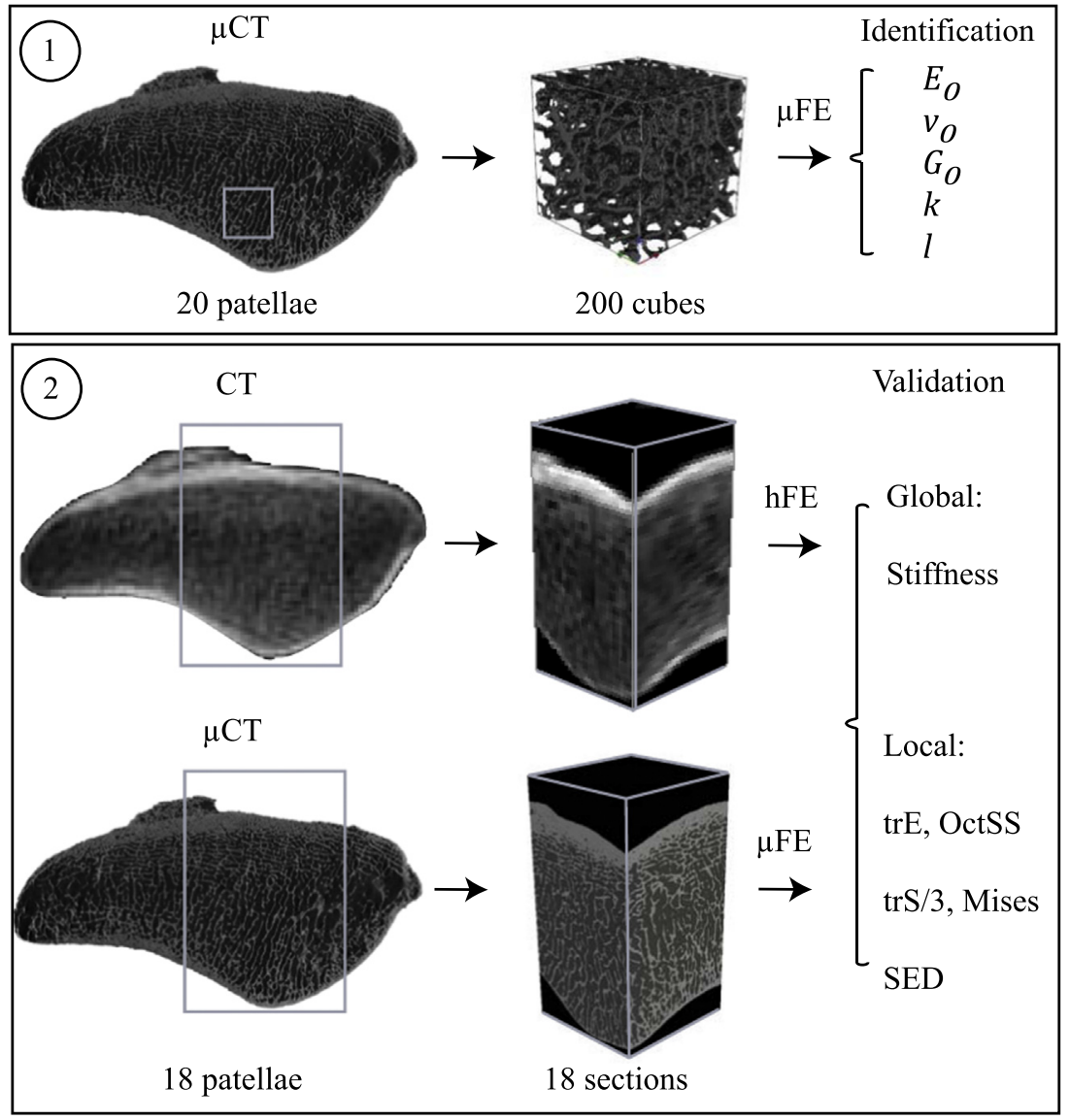

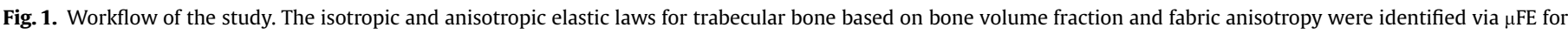

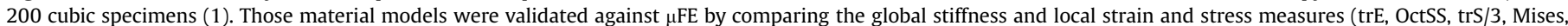
SED) of 18 patellar sections (2). 
orthotropic part was considered $\left(\mathbf{C}_{\mu F E}\right.$ and $\left.\mathbf{C}_{h F E}\right)$. The difference was estimated by

$N E_{h F E}=\frac{\left\|C_{\mu F E}-C_{h F E}\right\|}{\left\|C_{\mu F E}\right\|}$

In addition, the statistical significance between error induced by isotropic and anisotropic assumption was estimated with paired $t$-test. For local comparison, ten cubic ( $5.4 \mathrm{~mm}$ side) regions of interest (ROI) were extracted from each section. The size of the cubes was chosen to be consistent with the homogenization during the identification study. The components of strain and stress tensors were averaged on these ROIs. Volumetric strain (trE), octahedral shear strain (OctSS), volumetric stress (trS/3), von Mises stress (Mises), and strain energy density (SED) were compared. The hFE predictions were quantified with adjusted correlation coefficient $\left(r_{a d j}^{2}\right)$, concordance correlation coefficient (ccc) (Lin, 1989), root mean square error (RMSE) and $p$ value. The statistical significance between the correlations was analyzed with Williams formula proposed by (Steiger, 1980). Significance level was set to $95 \%(p<0.05)$ for all statistical analyses. The analysis of OctSS, Mises and SED followed a logarithmic transformation. Global stiffness was obtained by postprocessing functions of Medtool. Strain and stress tensors in integration points of ROI were extracted from $\mu \mathrm{FE}$ results with Paraview (paraview.org), and with python script (Python 2.6.6) from the hFE results. ROIs were extracted from 10 patellar sections since 8 of 18 sections could not be loaded in Paraview, probably due to large (up to $42 \mathrm{~GB}$ ) file sizes.

\section{Results}

The average bone volume fraction $\rho$ of 200 cubes was $0.29 \pm 0.11$. Eigenvalues of the fabric tensor were $m_{1}=0.77 \pm 0.07$, $m_{2}=0.99 \pm 0.06, m_{3}=1.23 \pm 0.09$. DA was $1.62 \pm 0.23$. The norm error $N E_{\text {aniso-ortho }}$ was $6.15 \pm 2.92 \%$. Both anisotropic and isotropic homogenized laws were strongly correlated to the $\mu \mathrm{FE}$ reference (Table 1).

The validation was conducted on 18 sections. Both anisotropic and isotropic hFE showed good match to $\mu \mathrm{FE}$ reference (Table 1). The anisotropic norm error $\mathrm{NE}_{\mathrm{hFE}}$ was lower $(13 \pm 5 \%)$ than the isotropic one ( $18 \pm 6 \%)$. The isotropic norm error was statistically significantly different from anisotropic norm error $(p=0.0009)$.

Local comparison was conducted on 100 cubic ROIs. The correlations of $\mathrm{hFE}$ against $\mu \mathrm{FE}$ for volumetric strain and stress were strong for anisotropic and isotropic models, but better for anisotropic one (Table 1). For OctSS, SED and Mises, anisotropic predictions also provided higher correlation coefficients, slopes of regression line closer to unit and lower RMS errors. The correlations were statistically significantly different $(p<0.001)$. The strain and stress distribution of hFE model was visually consistent with $\mu \mathrm{FE}$ reference (Fig. 2).

\section{Discussion}

Currently, literature is lacking validated material law for the patellar bone that can be implemented in hFE models. Such models can be used for numerical predictions of patellar strain in order to better understand patellar pathologies such as fracture after total knee arthroplasty (Fitzpatrick et al., 2011, 2013) or anterior knee pain (Ho et al., 2014). In this study we identified and validated anisotropic and isotropic material models for patellar trabecular bone by means of $\mu \mathrm{FE}$ analyses on 20 cadaveric patellae that can be further used in hFE models.

The average bone volume fraction $\rho$ of the extracted cubes was consistent with the reported values (Lammentausta et al., 2006; Raux et al., 1975). The structure of the patellar trabecular was found to be closer to orthotropic rather than isotropic or transverse isotropic. The low error caused by orthotropic approximation confirmed this observation. The average degree of anisotropy was in the range of other anatomical zones (femur, radius, and vertebra) (Gross et al., 2013), but no data for the patellar bone was found for comparison. The identified model parameters were also consistent with the literature (Gross et al., 2013), but higher exponential constants were obtained. It suggests that higher stiffness at low bone volume fraction zones and lower stiffness at higher bone volume fraction zones will be assigned to the patellar hFE if material parameters from other anatomical sites are used.

For validation of the identified elastic laws, the global stiffness and local strain and stress invariants of the bone sections modeled as hFE was compared to its $\mu \mathrm{FE}$ equivalent. The isotropic hFE was based on CT images since currently it is a gold standard in clinical application. The analyzed invariants were chosen as the common descriptors of bone strain and stress state that can be linked with bone damage. Both models showed highly significant correlation to $\mu \mathrm{FE}$ predictions, however, as expected, accounting for the trabecular fabric improved the correlation of the model against the $\mu \mathrm{FE}$ analyses (Gross et al., 2013; Maquer et al., 2015). Even though the anisotropic model better predicted the $\mu \mathrm{FE}$ global stiffness, it

Table 1

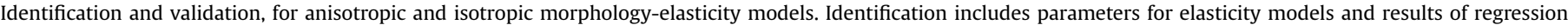

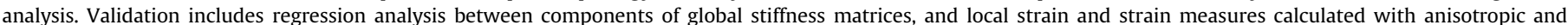
isotropic hFE models $(x)$ against $\mu$ FE reference $(y)$.

\begin{tabular}{|c|c|c|c|c|c|c|c|c|c|c|}
\hline \multicolumn{11}{|c|}{ Identification } \\
\hline & $E_{0}(\mathrm{MPa})$ & $\nu_{\mathbf{0}}$ & $G_{0}(\mathrm{MPa})$ & $\boldsymbol{k}$ & $\boldsymbol{l}$ & Regression & $r_{a d j}^{2}$ & ccc & RMSE & $p$-Value \\
\hline Anisotropy & 12723.1 & 0.24 & 4224.6 & 2.1 & 1.02 & $y=0.949 x+0.316$ & 0.95 & 0.97 & 0.20 & $<0.001$ \\
\hline Isotropy & 11035.9 & 0.26 & 4395.1 & 2.13 & - & $y=0.838 x+1.000$ & 0.85 & 0.92 & 0.32 & $<0.001$ \\
\hline \multicolumn{11}{|c|}{ Validation } \\
\hline & & & & & & Regression & $r_{a d j}^{2}$ & ccc & RMSE & $p$-Value \\
\hline \multirow[t]{6}{*}{ Anisotropy } & \multicolumn{5}{|c|}{ Global stiffness matrices } & $y=1.070 x-0.641$ & 0.96 & 0.96 & 0.16 & $<0.001$ \\
\hline & \multicolumn{5}{|c|}{ Volumetric strain (trE) } & $y=0.961 x+1 \mathrm{e}^{-5}$ & 0.98 & 0.99 & $8 e^{-5}$ & $<0.001$ \\
\hline & \multicolumn{5}{|c|}{ Octahedral shear strain (OctSS) } & $y=0.988 x-0.083$ & 0.84 & 0.91 & 0.07 & $<0.001$ \\
\hline & \multicolumn{5}{|c|}{ Volumetric stress (trS/3) } & $y=0.972 x+0.013$ & 0.98 & 0.98 & 0.08 & $<0.001$ \\
\hline & \multicolumn{5}{|c|}{ Von Mises stress (Mises) } & $y=0.913 x-0.001$ & 0.96 & 0.98 & 0.19 & $<0.001$ \\
\hline & \multicolumn{5}{|c|}{ Strain energy density (SED) } & $y=0.913 x-0.662$ & 0.98 & 0.98 & 0.17 & $<0.001$ \\
\hline \multirow[t]{6}{*}{ Isotropy } & \multicolumn{5}{|c|}{ Global stiffness matrices } & $y=0.978 x+0.094$ & 0.93 & 0.96 & 0.21 & $<0.001$ \\
\hline & \multicolumn{5}{|c|}{ Volumetric strain (trE) } & $y=0.962 x+2 e^{-5}$ & 0.96 & 0.98 & $1 e^{-4}$ & $<0.001$ \\
\hline & \multicolumn{5}{|c|}{ Octahedral shear strain (OctSS) } & $y=0.946 x-0.367$ & 0.71 & 0.84 & 0.10 & $<0.001$ \\
\hline & \multicolumn{5}{|c|}{ Volumetric stress (trS/3) } & $y=0.846 x+0.021$ & 0.94 & 0.96 & 0.16 & $<0.001$ \\
\hline & \multicolumn{5}{|c|}{ Von Mises stress (Mises) } & $y=0.792 x-0.112$ & 0.80 & 0.79 & 0.33 & $<0.001$ \\
\hline & \multicolumn{5}{|c|}{ Strain energy density (SED) } & $y=0.835 x-1.316$ & 0.89 & 0.86 & 0.29 & $<0.001$ \\
\hline
\end{tabular}



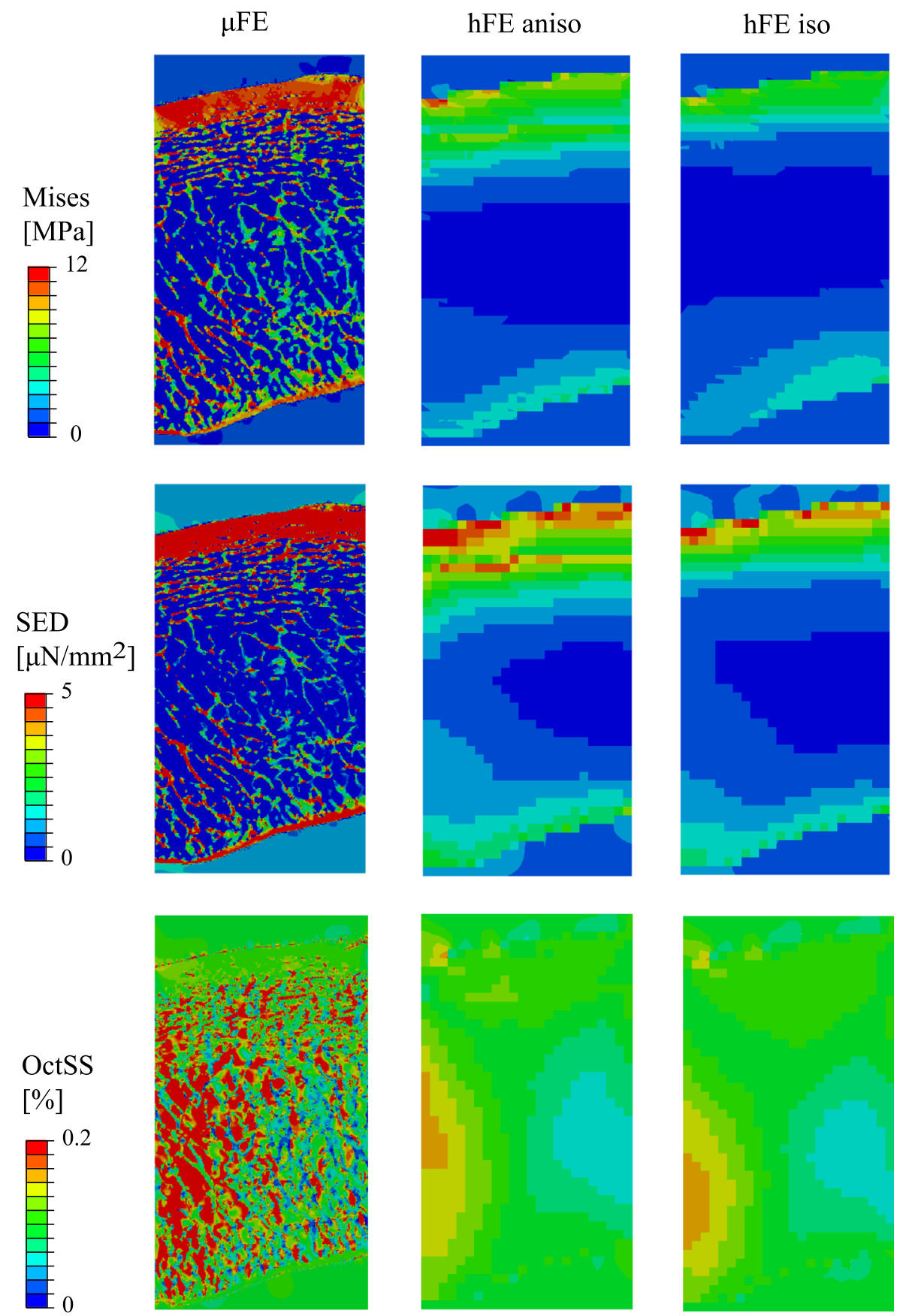

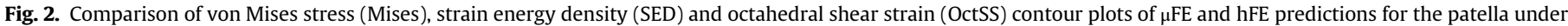
tension in superior-inferior direction (patellar cut in sagittal plane).

was still found slightly less stiff, possibly due to the lack of explicit cortex modeling (Pahr and Zysset, 2009). The highest variation between $\mu \mathrm{FE}$ and hFE stress predictions was found in cubes with low stress values caused by low bone volume fraction. These finding supports results of a similar study conducted on the proximal femur (Hazrati Marangalou et al., 2012). The correlation of the local strain predictions was lower than those of stress predictions most probably due to the lower variation of strain values.

Several limitations of the study should be mentioned. The identification was conducted on bone cubes using kinematic uniform boundary conditions that tend to overestimate bone effective stiffness (Hazanov and Huet, 1994). The bone tissue was assumed homogeneous and isotropic material, but it was demonstrated previously that heterogeneous tissue mineralization has only a minor effect on apparent trabecular bone elastic properties (Gross et al., 2012). The material model validation with six canonical loading cases of patellar cuboid sections allowed controlled boundary conditions and easy result interpretation (van Rietbergen and Ito, 2015; Zysset et al., 2013). However, predictions of anisotropic and isotropic models should be further compared for a whole patella under more physiological loading conditions. The importance of modeling anisotropy could be further emphasized in such case.

In conclusion, an anisotropic and an isotropic morphologyelasticity model for patellar trabecular bone were identified and validated. When high-resolution images are available, the anisotropic material parameters can be assigned to hFE models, assuming direct access to bone volume fraction and fabric tensor 
from images. When only low-resolution images are available, such as clinical CT scans, the isotropic model is a reasonable alternative. The anisotropic model might still be applicable by estimating anisotropy with recently proposed approaches, such as database approach or $\mu \mathrm{CT}$ template registration (Marangalou et al., 2013; Taghizadeh et al., 2016).

\section{Conflict of interest}

None of the authors has any conflict of interest.

\section{Acknowledgments}

This study was supported by the "Fondation de soutien à la recherche dans le domaine de l'orthopédie-traumatologie".

\section{References}

Cowin, S.C., 1985. The relationship between the elasticity tensor and the fabric tensor. Mech. Mater. 4, 137-147.

Fitzpatrick, C.K., Baldwin, M.A., Ali, A.A., Laz, P.J., Rullkoetter, P.J., 2011. Comparison of patellar bone strain in the natural and implanted knee during simulated deep flexion. J. Orthop. Res.: Off. Publ. Orthop. Res. Soc. 29, 232-239.

Fitzpatrick, C.K., Kim, R.H., Ali, A.A., Smoger, L.M., Rullkoetter, P.J., 2013. Effects of resection thickness on mechanics of resurfaced patellae. J. Biomech. 46, $1568-1575$.

Gross, T., Pahr, D.H., Zysset, P.K., 2013. Morphology-elasticity relationships using decreasing fabric information of human trabecular bone from three major anatomical locations. Biomech. Model. Mechanobiol. 12, 793-800.

Gross, T., Pahr, D.H., Peyrin, F., Zysset, P.K., 2012. Mineral heterogeneity has a minor influence on the apparent elastic properties of human cancellous bone: a SRmuCT-based finite element study. Comput. Methods Biomech. Biomed. Eng. $15,1137-1144$.

Hazanov, S., Huet, C., 1994. Order relationships for boundary-conditions effect in heterogeneous bodies smaller than the representative volume. J. Mech. Phys. Solids 42, 1995-2011.

Hazrati Marangalou, J., Ito, K., van Rietbergen, B., 2012. A new approach to determine the accuracy of morphology-elasticity relationships in continuum FE analyses of human proximal femur. J. Biomech. 45, 2884-2892.

Hildebrand, T., Laib, A., Muller, R., Dequeker, J., Ruegsegger, P., 1999. Direct threedimensional morphometric analysis of human cancellous bone: microstructural data from spine, femur, iliac crest, and calcaneus. J. Bone Mineral. Res.: Off. J. Am. Soc. Bone Mineral. Res. 14, 1167-1174.

Ho, K.Y., Keyak, J.H., Powers, C.M., 2014. Comparison of patella bone strain between females with and without patellofemoral pain: a finite element analysis study. J. Biomech. 47, 230-236.
Laib, A., Hauselmann, H.J., Ruegsegger, P., 1998. In vivo high resolution 3D-QCT of the human forearm. Technol. Health Care: Off. J. Eur. Soc. Eng. Med. 6, 329-337.

Lammentausta, E., Hakulinen, M.A., Jurvelin, J.S., Nieminen, M.T., 2006. Prediction of mechanical properties of trabecular bone using quantitative MRI. Phys. Med. Biol. 51, 6187-6198.

Latypova, A., Arami, A., Becce, F., Jolles-Haeberli, B., Aminian, K., Pioletti, D.P., Terrier, A., 2016. A patient-specific model of total knee arthroplasty to estimate patellar strain: a case study. Clin. Biomech. 32, 212-219.

Lin, L.I., 1989. A concordance correlation coefficient to evaluate reproducibility. Biometrics 45, 255-268.

Maquer, G., Musy, S.N., Wandel, J., Gross, T., Zysset, P.K., 2015. Bone volume fraction and fabric anisotropy are better determinants of trabecular bone stiffness than other morphological variables. J. Bone Mineral. Res. 30, 1000-1008.

Marangalou, J.H., Ito, K., Cataldi, M., Taddei, F., van Rietbergen, B., 2013. A novel approach to estimate trabecular bone anisotropy using a database approach. J. Biomech. 46, 2356-2362.

Pahr, D.H., Zysset, P.K., 2008. Influence of boundary conditions on computed apparent elastic properties of cancellous bone. Biomech. Model. Mechanobiol. 7, 463-476.

Pahr, D.H., Zysset, P.K., 2009. A comparison of enhanced continuum FE with micro FE models of human vertebral bodies. J. Biomech. 42, 455-462.

Pistoia, W., van Rietbergen, B., Lochmuller, E.M., Lill, C.A., Eckstein, F., Ruegsegger, P., 2002. Estimation of distal radius failure load with micro-finite element analysis models based on three-dimensional peripheral quantitative computed tomography images. Bone 30, 842-848.

Raux, P., Townsend, P.R., Miegel, R., Rose, R.M., Radin, E.L., 1975. Trabecular architecture of the human patella. J. Biomech. 8, 1-7.

Ridler, T.W., Calvard, S., 1978. Picture thresholding using an iterative selection method. IEEE Trans. Syst. Man Cybern. 8, 630-632.

Steiger, J.H., 1980. Tests for comparing elements of a correlation matrix. Psychol. Bull. 87, 245-251.

Taghizadeh, E., Reyes, M., Zysset, P., Latypova, A., Terrier, A., Buchler, P., 2016. Biomechanical role of bone anisotropy estimated on clinical CT scans by image registration. Ann. Biomed. Eng.

Takahashi, A., Sano, H., Ohnuma, M., Kashiwaba, M., Chiba, D., Kamimura, M., Sugita, T., Itoi, E., 2012. Patellar morphology and femoral component geometry influence patellofemoral contact stress in total knee arthroplasty without patellar resurfacing. Knee Surg. Sport. Traumatol. Arthrosc.: Off. J. ESSKA 20, 1787-1795.

van Rietbergen, B., Ito, K., 2015. A survey of micro-finite element analysis for clinical assessment of bone strength: the first decade. J. Biomech. 48, 832-841.

Whitehouse, W.J., 1974. The quantitative morphology of anisotropic trabecular bone. J. Microsc. 101, 153-168.

Wolfram, U., Wilke, H.J., Zysset, P.K., 2010. Valid micro finite element models of vertebral trabecular bone can be obtained using tissue properties measured with nanoindentation under wet conditions. J. Biomech. 43, 1731-1737.

Zysset, P.K., 2003. A review of morphology-elasticity relationships in human trabecular bone: theories and experiments. J. Biomech. 36, 1469-1485.

Zysset, P.K., Curnier, A., 1995. An alternative model for anisotropic elasticity based on fabric tensors. Mech. Mater. 21, 243-250.

Zysset, P.K., Dall'ara, E., Varga, P., Pahr, D.H., 2013. Finite element analysis for prediction of bone strength. BoneKEy Rep. 2, 386. 